\title{
Indirect evidence for short period magnetic cycles in W UMa stars
}

\section{Period analysis of five overcontact systems}

\author{
T. Borkovits ${ }^{1}$, M. M. Elkhateeb ${ }^{2}$, Sz. Csizmadia ${ }^{3}$, J. Nuspl ${ }^{3}$, I. B. Bíró1 ${ }^{1}$, T. Hegedüs ${ }^{1}$, and R. Csorvási ${ }^{4,5}$ \\ 1 Baja Astronomical Observatory of Bács-Kiskun County, 6500 Baja, Szegedi út, Kt. 766, Hungary \\ e-mail: borko@alcyone.bajaobs.hu \\ 2 Dept. of Astronomy, National Research Institute of Astronomy and Geophysics (NRIAG), Helwan, Cairo, Egypt \\ 3 Konkoly Observatory of the Hungarian Academy of Sciences, 1525 Budapest, Pf. 67, Hungary \\ ${ }^{4}$ Department of Optics \& Quantum Electronics \& Astronomical Observatory, University of Szeged, \\ 6701 Szeged, Pf. 406, Hungary \\ 5 Guest Observer at Piszkéstető Station, Konkoly Observatory, Hungary
}

Received 2 February 2005 / Accepted 24 May 2005

\section{ABSTRACT}

Complex period variations of five W UMa type binaries (AB And, OO Aql, DK Cyg, V566 Oph, U Peg) were investigated by analyzing their $\mathrm{O}-\mathrm{C}$ diagrams, and several common features were found. Four of the five systems show secular period variations at a constant rate on the order of $\left|\dot{P}_{\text {sec }} / P\right| \sim 10^{-7} \mathrm{yr}^{-1}$. In the case of AB And, OO Aql, and U Peg a high-amplitude, nearly one-century long quasi-sinusoidal pattern was also found. It might be explained as light-time effect, or by some magnetic phenomena, although the mathematical, and consequently the physical, parameters of these fits are very problematic, as the obtained periods are very close to the length of the total data range. The most interesting feature of the studied O-C diagrams is a low amplitude $\left(\sim 2-4 \times 10^{-3}\right.$ d) modulation with a period around 18-20 yr in four of the five cases. This phenomenon might be indirect evidence of some magnetic cycle in late-type overcontact binaries as an analog to the observed activity cycles in RS CVn systems.

Key words. binaries: close - binaries: eclipsing - stars: activity - stars: general

\section{Introduction}

The moments of minima are one of the most fundamental observables of eclipsing binary systems and, due to their relatively easy measurement, a huge pile of data of this type was collected by observers during the last century. However, so far there is no a commonly accepted and straightforward interpretation of the $\mathrm{O}-\mathrm{C}$ diagrams constructed from them. Additional difficulties arise when one tries to disentangle and identify the physical mechanisms causing the observed period variations although this would be the aim. For example, in spite of the fact that the period variations are common features of contact binary stars (Kreiner 1977; Maceroni \& van't Veer 1996), their origin is not well understood yet.

These observed variations can be classified into two subsets: (i) in the first part are the apparent period variations due to light-time effect (LITE) caused by a distant third or further body ${ }^{1}$, and (ii) the second part comprises the inherent physical period variations. The latter may also be of various types,

${ }_{1}$ The other fundamental class of the apparent period variations, namely, the effect of the apsidal motion (AM) is not considered here due to the circular orbit of the systems. e.g. caused by the evolution of the system, mechanical and/or thermodynamical effects, or due to variable magnetic activity. Of course, a mixture of the different sources can be active at the same time, generating a very complex variation in the orbital period.

In respect to the involved time scales we can distinguish the long-term variations (on a nuclear or thermal time scale) from the short-term variations characterised by a decade-long cycle length. In our recent study we focus on the short-term orbital period variations of several W UMa systems, because the real $\mathrm{O}-\mathrm{C}$ observations can reveal only these effects and the longterm ones require a different (statistical) approach.

\section{Long-term effects}

Recently, the internal structure and evolution of contact W UMa systems are not well-known or else are poorly understood. The evolution theories of these binary stars yield secular as well as shorter time scale quasi-cyclical orbital period variations. Although these theories suffer from a lot of problems their predictions can be used as guidelines (Kreiner et al. 2003; Kähler 2004) to better understand the behaviour of these systems. 
All the currently available theories predict a secular orbital period increase of such systems caused by mass transfer between the components. Some theories, e.g. the Thermal Relaxation Oscillation (TRO) (Lucy 1976; Webbink 1976) or discontinuity (DSC) theory (Lubow \& Shu 1977), give rise to different periodic and secular variations of the orbital period and of the observable light curves. However, Mochnacki (1981); Kähler (1986), as well as Hazlehurst (2001) showed that none of these theories agree with the observations or seem to violate the second law of thermodynamics. The Angular Momentum Loss (AML) theories are based on the assumption of the angular momentum loss via magnetic breaking on stellar winds (van't Veer 1979) or on the magnetic fields of the companion star (Mochnacki 1981), or even on the simple mass and angular momentum loss via stellar wind (van't Veer \& Maceroni 1989).

Kähler (2004) integrated the evolutionary equation of a model contact system and found that the systems are oscillating both with and without losing their contact configuration. During these oscillations the period shows (quasi-)periodic variations on a time scale of several million years. The origin of this period variation is once again mass transfer between the components. Note that Qian (2001a) suggested that systems with $q<0.4$ show secular period decrease and systems with $q>0.4$ secular period increase ( $q$ is the mass ratio), an effect that - if real - has not yet been interpreted theoretically.

Independent of any uncertainties in them, all of these theories predict the period variations on a time-scale comparable to the nuclear evolution time-scale of the primary components i.e. some billion years which is far from what is observed in the $\mathrm{O}-\mathrm{C}$ diagrams.

\section{Short-term effects}

Short-term effects are the most exciting ones for us because they can be studied through the observed times of minima and the $\mathrm{O}-\mathrm{C}$ diagrams.

The LITE that cause apparent orbital period variations was studied in many contact binary systems, and one of the most recent extended studies was published in Borkovits \& Hegedüs (1996), also includes 18 Algol and contact systems. They found that only four of the 18 cases studied might have a third (or fourth) companion with high probability. Remarkably two of the studied five W UMa systems, namely AB And and AK Her, could have a third component compared to the two Algol type systems from the thirteen ones considered.

However, Hendry \& Mochnacki (1998) found that AK Her does not show spectroscopic evidence for a third companion in the system. Note that this star has a wider, visual companion at a separation of $\rho=4.7$, nevertheless this companion cannot be responsible for the decades-scale cyclic variation of the O-C. Since the time coverage of their observations was only a few days, the gamma velocity was not changed, while the lack of the third body's spectroscopic line can be explained by a low luminosity object (e.g. a white dwarf). They also found from spectroscopy that SW Lac and V502 Oph have a third body, although the $\mathrm{O}-\mathrm{C}$ diagrams did not show their presence clearly.
These facts might be explained by a high inclination effect. This contradiction between the $\mathrm{O}-\mathrm{C}$ diagrams and the spectroscopic observations has not been solved yet.

Note that the light-time effect explanations of the $\mathrm{O}-\mathrm{C}$ diagrams are uncertain in those cases when less than one revolution of the third body could be observed till now. One important example for this latter statement can be the case of AK Herculis (Rovithis-Livaniou et al. 1999). At the same time, in the case of VW Cephei the period of the LITE orbit deduced from the $\mathrm{O}-\mathrm{C}$ diagram is in very good agreement with the astrometric observations (Hershey 1975; Heintz 1993), although the amplitude of the LITE and, consequently, the projected semimajor axis of the third body orbit was found to be inconsistent with these other measurements (see Kaszás et al. 1998). Nevertheless, independent of the possible reasons for this inconsistency, it seems evident that at least a significant part of the cyclic $\mathrm{O}-\mathrm{C}$ variation arises from the astrometrically, and interferometrically identified tertiary. Although it is clear that the LITE studies should be improved, it is not an easy task considering that the typical time-scale of the revolution period of the third body is as long as several decades or centuries. Thus, it should be emphasised that independent spectroscopic affirmations are inevitable before one can approve any LITE solution constructed from $\mathrm{O}-\mathrm{C}$ diagrams.

A further reason for period variations on a short time-scale can be the magnetic activity of the contact binary system. There are significant pieces of evidence for their magnetic activity, such as

(i) Doppler-tomography (Barnes et al. 2004; Hendry \& Mochnacki 2000; Maceroni et al. 1994);

(ii) O'Connell-effect and their spot-explanation in many systems;

(iii) their X-ray emission deviations (Stepien et al. 2001);

(iv) $H_{\alpha}$ emission in some $\mathrm{W}$ UMa systems (e.g. Hrivnak et al. 1995; Csizmadia et al. 2004).

From an extended study of the period variations of a sample containing roughly 100 Algol systems, Hall (1989) concluded that the spectral type of systems showing cyclic period variations is later than F5. This earlier hypothesis was supported during the last decade by several investigations.

Magnetic activity is a manifestation of strong magnetic fields present in the system, which interact with the matter of stars in plasma state and could change the inertia tensor of component(s) through this interaction. Since the inertia tensor is a part of the total angular momentum required to be constant, the variation in the magnetic field structure might influence the orbital period of the system in this way. This effect is known as "the interchange of the magnetic and kinetic energy" (Applegate 1992; Lanza \& Rodonó 2002). The timescale of this so-called Applegate-mechanism is several years or decades, and its amplitude may be as large as 0.006 days. This value is about $10-20$ times higher than the accuracy of one minimum time observation.

Other reasons for period variations can also be imagined, e.g. sudden mass exchange via large flares, gaining a planet, etc. Time-dependent variable long-scale current in the common 
convective envelope could also cause variable orbital periods, while all of these effects can affect the $\mathrm{O}-\mathrm{C}$ diagram.

Since now more minima observations are available than a decade ago and further theoretical calculations on the perturbational effects of a third body were carried out (e.g. Borkovits et al. 2003), we decided to improve and re-analyse the O-C diagrams of some eclipsing contact binary stars. In Sect. 2 we describe the method and in Sect. 3 we study these systems individually. The variations found in the $\mathrm{O}-\mathrm{C}$ diagrams are discussed in Sect. 4 and our conclusions can be found in Sect. 5 .

\section{General considerations on the analysis of the O-C diagrams}

Finding a common explanation of the possible complex behaviour of $\mathrm{O}-\mathrm{C}$ diagrams is not an easy task as it requires careful detailed analysis and modelling of the data set. It is well-known that the quite general model of $\mathrm{O}-\mathrm{C}$ data as the modulation of a secular (parabolic) trend with some periodic additional variations due to different physical effects can usually describe most of the observed $\mathrm{O}-\mathrm{C}$ diagrams. In this representation, the secular part is connected to long-term evolution effects (mainly to mass exchange between the components) and the periodic modulations can be connected to e.g. LITE caused by a distant third companion or, if no reasonable third-orbit solution can be given, then some quasi-periodic magnetic effects or repeated mass-exchange processes.

Nevertheless, there are only a few systems where the presence of a third companion has already been proved by independent measurements. In such systems (e.g. VW Cephei, XY Leonis), the tertiary components have a period several times shorter than the total observational interval of the eclipsing minima range, and these are clear cases of straightforward interpretation. Difficulty arises if the period of the cyclical term (if any) is close to or longer than the observational window, especially if any secular behaviour is also present. It is evident that an independent fitting of the secular parabolic term and sinusoidal terms - as was done in several earlier works - may give false results, and even a simultaneous fitting does not necessarily improve the situation. Instead of the detailed description of these problems, which are discussed and published elsewhere, we here give only two illustrations to these statements. We show that such a simultaneous fit in the case what is of already one simple abrupt period change can produce a reasonable - secular plus periodic - orbital period change solution.

In order to demonstrate this, we constructed different artificial times of minima data series. All of them have the following ephemeris: $\mathrm{MIN}_{\mathrm{I}}=2445000.0+0.4556788 \mathrm{E}$ for $E<0$, and $\mathrm{MIN}_{\mathrm{I}}=2445000.0+0.4556790 \mathrm{E}$ for $E>0$. The data were generated with a random accuracy of about 0.0008 days and with random epochs (i.e. random $E$ value). More accurate points than 0.0002 days were weighted as photoelectric data. Here we present two data series, one which was calculated on the symmetric interval $-80000<E<80000$, while the second one on the asymmetric one of $-110000<E<50000$.

The data series were analysed in a similar manner to the one described in Borkovits et al. (2002), i.e. after calculating the $\mathrm{O}-\mathrm{C}$ curve with a preliminary linear ephemeris, the final
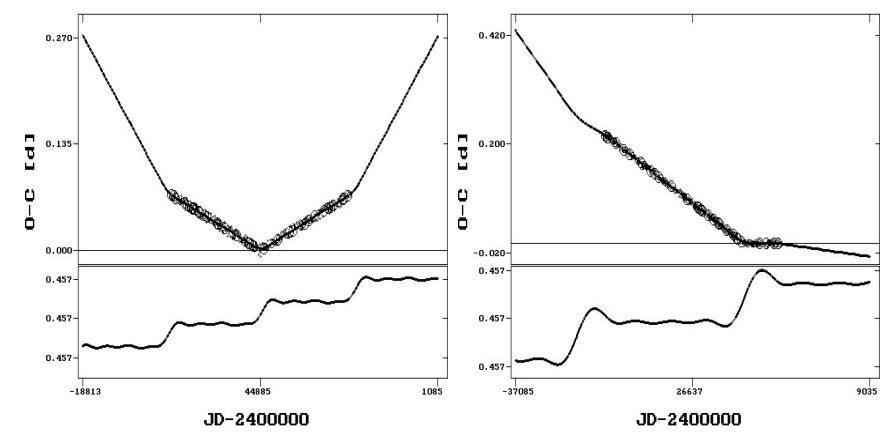

Fig. 1. The artificial $\mathrm{O}-\mathrm{C}$ curves with simultaneously fitted leastsquare parabolas and Fourier-curves (above) and the period curves (bottom). The plotted time-intervals are twice longer than the lengths of the data series.

representation of the $\mathrm{O}-\mathrm{C}$ was searched for by a weighted linear least-squares fit in the form:

$f=c_{0}+c_{1} E+c_{2} E^{2}+\sum_{i, j} a_{i j} \sin j v_{i} E+b_{i j} \cos j v_{i} E$,

where $0 \leq i \leq 5,1 \leq j \leq 4$. The frequencies $v_{i}$ were kept fixed during the individual LSQ runs, but an interval of frequencies was scanned and the best parameter set was selected according to the smallest $\chi^{2}$ test.

In the present cases $i=1, j=1 . .3$ were applied, e.g. one fundamental frequency; and its first two harmonics were used to obtain the very good fits, which are presented in Fig. 1. Furthermore, what is perhaps more suprising is that the ratio of the Fourier-coefficients, which belong to different harmonics, almost exactly satisfy the condition of a light-time orbit. Strictly speaking, we could find reasonable third body solutions in both cases, which were almost independent of whether they were calculated only from the $a_{1,2}, b_{1,2}$ coefficients (Kopal 1978, Chap. V), or the coefficients of the second harmonics (Borkovits \& Hegedüs 1996) were also included.

Of course, the false results can be understood considering that removing the parabolic trend from the data set the residual variations form a sawtooth-like periodic function, which can be represented quite well by some low order terms of its Fourierseries. On the other hand, the solutions were significantly different for the two curves, which sheds light on another problem of the simultaneous fitting. Namely, if only approximately one period is covered by the observations, the coefficient obtained for the quadratic term and the period of the periodic variation are strongly coupled, and also depend on the length of the data.

In what follows we analyse the $\mathrm{O}-\mathrm{C}$ curves of several contact binaries, some of which clearly demonstrate this latter statement. However, we have to emphasise that in our cases these uncertainties are connected only to the longer period variations but not to the shorter ones which are the main targets of our discussion here.

\section{Analysis of real systems}

We concentrated mainly on systems whose observation covers more than a half, or even a complete century. The most important parameters of the analysed five systems are listed in 
Table 1. Main parameters of the investigated stars. The spectral types are taken from SIMBAD Database ${ }^{\star}$.

\begin{tabular}{llllllllllll}
\hline \hline Name & $\mathrm{Sp}$ & $P$ & $\mathrm{~T}$ & $M_{1}$ & $M_{2}$ & $R_{1}$ & $R_{2}$ & $T_{1}$ & $T_{2}$ & $A$ & Refs. \\
\hline AB And & $G 5 V^{a}$ & 0.33 & $\mathrm{~W}$ & 0.60 & 1.04 & 0.78 & 1.03 & 5450 & 5798 & 2.37 & 1 \\
OO Aql & $G 5 V$ & 0.51 & $\mathrm{~A}$ & 1.04 & 0.88 & 1.39 & 1.29 & 5700 & 5560 & 3.33 & 2 \\
DK Cyg & $A 6 V$ & 0.47 & $\mathrm{~A}$ & 1.74 & 0.53 & 1.71 & 0.99 & 7351 & 7200 & 3.34 & 1 \\
V566 Oph & $F 4 V$ & 0.41 & $\mathrm{~A}$ & 1.56 & 0.41 & 1.51 & 0.86 & 6700 & 6618 & 2.91 & 3 \\
U Peg & $G 2 V$ & 0.37 & $\mathrm{~W}$ & 1.15 & 0.38 & 1.22 & 0.74 & 5860 & 5785 & 2.52 & 4 \\
\hline
\end{tabular}

${ }^{\star}$ http://simbad.u-strasbg.fr/sim-fid.pl

1: Baran et al. (2004); 2: Hrivnak (1989); 3: Niarchos et al. (1993); 4: Pribulla \& Vaňko (2002).

${ }^{a}$ More recently Pych et al. (2004) found a mean spectral type of G8V.

Table 1. The minima list of the five stars are not included in this paper due to their enormous size. Nevertheless, the total collection can be requested from the authors.

In several cases earlier observations are photographic or, more frequently, less accurate patrol measurements or visual observations. They were taken into account as well, at least at the first step of our analysis, but with different weights. We used four different weights: 1: visually observed minima; 2 : plate minima; 5: photographic normal minima; and 10: photoelectric observations (both photomultiplier tube and CCD).

\section{1. $A B$ Andromedae}

This overcontact binary that belongs to the $\mathrm{W}$ subclass has been very well observed since its discovery almost 80 years ago. A comprehensive analysis of the photometric data obtained between 1968 and 1995 was recently published by Djurašević et al. (2000), who concluded that the light-curve variations can be explained well by variation of the spot activity. Two detailed period investigations were carried out for this system in the last decade. Although two different methods were applied in those papers. Both Kalimeris et al. (1994) and Borkovits \& Hegedüs (1996) detected the combination of secular period variation and cyclic period change with a period which is close to the length of the observational interval. This fact makes it especially interesting to carry out a new $\mathrm{O}-\mathrm{C}$ study with an extended data series which is longer by about 10 years.

The analysed O-C data now cover 37181 days from HJD 2416103 to HJD 2453284 . As the photographic and photoelectric data cover all the interval well, apart from the approx. 6000 day-long gap after the first three data, we omitted the visual data in our analysis. A weighted LSQ fit of Eq. (1), with one fundamental frequency and with its first harmonic, resulted in a very reasonable fit. Nevertheless, after subtraction of this solution we found a second, small-amplitude quasiperiodic modulation with a period of $P_{\text {mod }} \approx 7000$ days. In order to investigate this possible modulation, we repeated our LSQ fitting procedure with two fundamental frequencies. At the smaller frequency (i.e. longer period) we also took the first harmonic into account, while at the higher frequency we did not fit any harmonics. Our final solution can be seen in Figs. 2 and 3. Supposing that the longer period periodic term arises

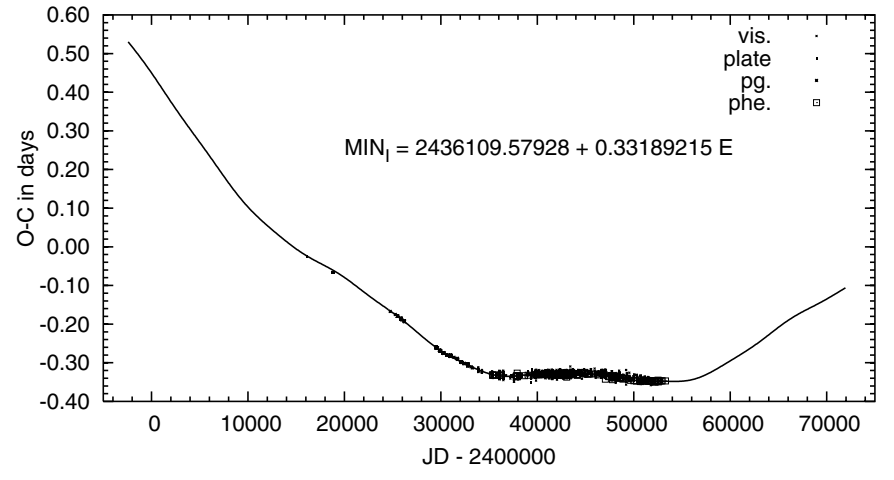

Fig. 2. The O-C curve of the eclipsing binary AB And, with the linear ephemeris taken from the GCVS (Kholopov et al. 1998) (above), and the simultaneous fit with two fundamental frequencies. We plotted the visual points, too, although these were discarded from the fitting procedure (see text for details).

from a light-time orbit, the orbital elements of the wide orbit of the binary around the centre of mass of the hierarchical triple system can be calculated easily. The results are listed in Table 2. Nevertheless, the calculated minimal mass of the hypothetical tertiary suggests that, if the tertiary is not a degenerate object, it has to produce a significant amount of third light, which should be observed. In contradiction to this statement, none of the previous light-curve solutions (at least those known for us) contains any third light. On the other hand, we have to keep in mind that, due to the complex effect of the individual fitting parameters on the light curve solutions of contact eclipsing binaries, one can obtain similarly satisfactory approximation of the same problem both with and without assuming third light; thus it would be necessary to reanalyse the light curves of $\mathrm{AB}$ And with third light. Furthermore, slight indirect evidence for the motion of the binary in a triple system arises from the comparison of the systemic velocity $\left(V_{0}\right)$ of the system in the latest two radial velocity surveys. While Hrivnak (1988) obtained $V_{0}=-24.6 \pm 0.9 \mathrm{~km} \mathrm{~s}^{-1}$ from the JD $2445886-2446285$ measurements, the most recent study of Pych et al. (2004) around JD 2452500 resulted in $V_{0}=-27.5 \pm 0.7 \mathrm{~km} \mathrm{~s}^{-1}$. In Fig. 4 we plotted the calculated systemic radial velocity variation of the system due to the revolution around the centre of mass of the triple system. As can be seen, our solution suggests a $\Delta V_{0} \approx-1 \mathrm{~km} \mathrm{~s}^{-1}$ variation between the above-mentioned two sets of observations, which is not all that far from the measured difference. Another possibility is to explain the cyclic variation by magnetic activity (see Sect. 4.3).

\section{2. $O 0$ Aquilae}

This high mass-ratio A-type overcontact binary was discovered by Hoffleit (1932). It belongs to the small group of longer period contact binaries which are supposed to have evolved into the contact phase in the (astronomically) near past (see e.g. Hrivnak et al. 2001, and references therein). Spectroscopic study by Hrivnak et al. (2001) revealed strong $\mathrm{Mg}$ II $\mathrm{h}$ and $\mathrm{k}$ emission lines varying with time, as clear evidence of chromospheric activity, although they also pointed 
Table 2. LITE solution for AB And.

\begin{tabular}{lll}
\hline \hline $\mathrm{MIN}_{\mathrm{I}}=2436109.265216(2)+0.3318897(25) E+3.99(1) \times 10^{-11} E^{2}$ \\
\hline$P^{\prime}$ & $($ day $)$ & 22742 \\
$e^{\prime}$ & & $0.10(1)$ \\
$\omega^{\prime}$ & $\left({ }^{\circ}\right)$ & $315(7)$ \\
$\tau^{\prime}$ & $(\mathrm{HJD})$ & $2436358(448)$ \\
$a_{12} \sin i^{\prime}$ & $\mathrm{AU}$ & $3.93(4)$ \\
$f\left(m_{3}\right)$ & $M_{\odot}$ & $0.0158(5)$ \\
$K_{12}$ & $\mathrm{~km} \mathrm{~s}^{-1}$ & 1.90 \\
\hline$m_{3}\left(M_{\odot}\right)$ & $i^{\prime}=90^{\circ}$ & 0.40 \\
& $i^{\prime}=60^{\circ}$ & 0.48 \\
& $i^{\prime}=30^{\circ}$ & 0.95 \\
\hline
\end{tabular}
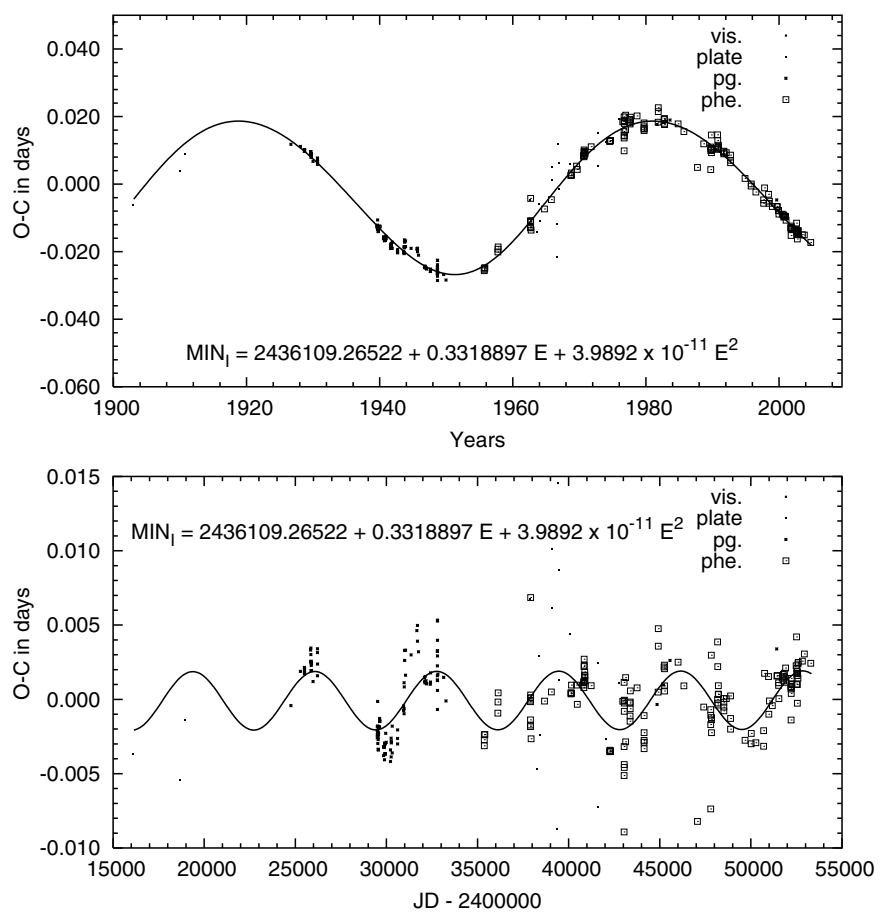

Fig. 3. Top: the LITE fit with the period $P^{\prime}=22742$ days. In order to see it better we removed the parabolic term, as well as the shorter period sinusoidal component. Bottom: the short term $\left(P_{\bmod }=6695\right.$ days $)$ modulation on the $\mathrm{O}-\mathrm{C}$ curve. For the better seeing we removed the other components of our final solution.

out that OO Aql is a less active system than other W UMa-type ones. The most detailed period study in the recent literature was published by Demircan \& Gürol (1996). They concluded that the most probable two scenarios for the large structure period variation were an abrupt period jump with $\Delta P \approx-0.6 \mathrm{~s}$ around 1963 or a sinusoidal variation with a period of $P^{\prime} \approx$ $90 \mathrm{yr}$, although this second statement was quite ambiguous, as the total curve covered only one maximum without any minima. They also mentioned the possibility of a parabolic representation. Nevertheless, in the present evolutionary state of the system, it could be expected that the binary evolves toward a smaller mass-ratio state, which should have resulted in an increasing period as was clearly in contradiction with

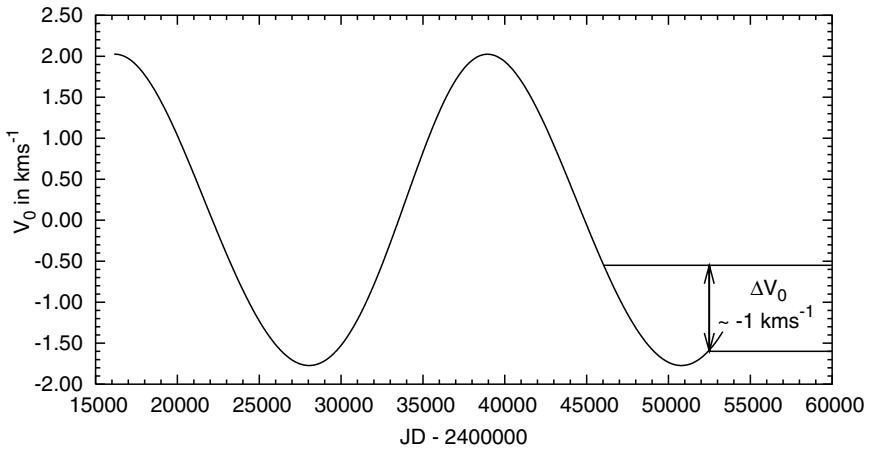

Fig. 4. The calculated systemic radial velocity $\left(V_{0}\right)$ variation of $\mathrm{AB}$ And according to the LITE solution listed in Table 2. We marked the positions of the radial velocity measurements of Hrivnak (1988) and Pych et al. (2004). See text for details.

the 1995 state. Demircan \& Gürol (1996) also investigated the presence of shorter, small amplitude fluctuations and concluded that these variations did not show simple periodic nature.

Since this aforementioned study, the picture has changed significantly. Around 1996 the period of the system also varied (see top panel in Fig. 5). Our O-C data extend on a 25919 daylong interval between HJD 2426892 and 2452811 . Although it cannot be excluded that this is a second abrupt period change, we may also suspect that the large amplitude sinusoidal variation reached its minimum. Consequently, we applied our fit with two fundamental periods: one (with its first harmonic) for the long period sinusoidal variation, and one for the short period fluctuations, which clearly reveals periodic behaviour in contrast to the statement of the previous authors. The simultaneous fit is plotted in the top panel of Fig. 5, while in the bottom panel we illustrate the periodic nature of $\mathrm{O}-\mathrm{C}$ lacking long period terms. Assuming that the $P^{\prime} \approx 75 \mathrm{yr}$ variation arises from the presence of a more distant tertiary component, the orbital elements of the binary in the triple system are listed in Table 3. As seen there, the minimal mass of the tertiary is again so large that (if not a degenerate object) it should be seen via the light curve with significant third light contribution, as well as via its spectral lines. Nevertheless, the previous light-curve studies give clear indirect evidence against the presence of any significant third light; namely, that the inclination of the system without the supposition of any third light was found to be close to $i=90^{\circ}$ (Hrivnak 1989; Djurašević \& Erkapić 1999). As the third light reduces the depth of the light minima, a given depth could be reproduced in its presence only by a larger inclination value than in the case of no third light, which would be physically unrealistic for the present star. Consequently, even if the 75 year-long periodic amplitude is real, an alternate explanation is needed for it.

\subsection{DK Cygni}

Although the discovery of this A-type overcontact binary was reported in the same paper (Guthnick \& Prager 1927) than as was the above-mentioned AB And, and their brightness is also similar, this system was less popular with the observers. Paparó et al. (1985) found a continuous period increase in the system, 

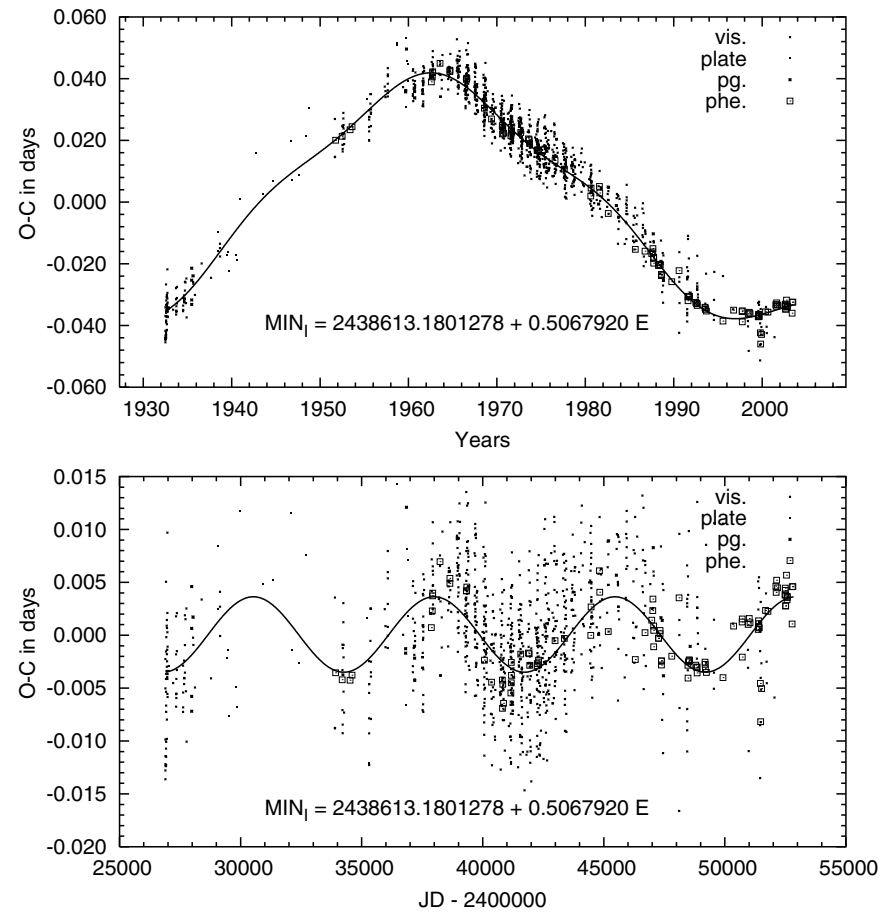

Fig. 5. The O-C curve of the eclipsing binary OO Aql with the calculated linear ephemeris and the simultaneous fit with two fundamental frequencies (above), and the smaller period fluctuations after the subtraction of the longer period curve. The Fourier-fits were carried out simultaneously.

Table 3. LITE solution for OO Aql.

\begin{tabular}{lll}
\hline \hline $\mathrm{MIN}_{\mathrm{I}}=$ & $24438613.18013(1)+0.5067920(14) E$ \\
\hline$P^{\prime}$ & $($ day $)$ & 27273 \\
$e^{\prime}$ & & $0.06(1)$ \\
$\omega^{\prime}$ & $\left({ }^{\circ}\right)$ & $23(13)$ \\
$\tau^{\prime}$ & $(\mathrm{HJD})$ & $2432894(970)$ \\
$a_{12} \sin i^{\prime}$ & $\mathrm{AU}$ & $6.56(5)$ \\
$f\left(m_{3}\right)$ & $M_{\odot}$ & $0.05078(11)$ \\
$K_{12}$ & $\mathrm{~km} \mathrm{~s}^{-1}$ & 2.62 \\
\hline$m_{3}\left(M_{\odot}\right)$ & $i^{\prime}=90^{\circ}$ & 0.70 \\
& $i^{\prime}=60^{\circ}$ & 0.84 \\
& $i^{\prime}=30^{\circ}$ & 1.77 \\
\hline
\end{tabular}

which was confirmed by Wolf et al. (2000). No spot activity was observed in this system as a survey of the literature showed.

The collected times of minima data in our analysis cover 28542 days from HJD 2424760 to HJD 2453302 . In the analysis we also used seven recently obtained unpublished minima, listed in Table 4. Omitting the visual data, a weighted LSQ fit resulted in the following parabolic ephemeris (see Fig. 6):

$\mathrm{MIN}_{\mathrm{I}}=2451000.1031+0.470693909 E+5.862 \times 10^{-11} E^{2},(2)$

which is in good agreement with the previous results. Despite this fact, we think that it is not without benefit to reanalyse the $\mathrm{O}-\mathrm{C}$ of this system, as well as any other eclipsing
Table 4. New times of minima of DK Cyg observed by R. Csorvási with CCD cameras mounted on the 40-cm Cassegrain telescope at Szeged Observatory, and the 90-cm Schmidt telescope at Piszkéstető Mountain Station of Konkoly Observatory.

\begin{tabular}{lll}
\hline \hline HJD & Error & Type \\
\hline 2453223.4286 & $\pm 0.0011 \mathrm{~s}$ \\
2453228.3681 & $\pm 0.0008 \mathrm{p}$ \\
2453246.4950 & $\pm 0.0013 \mathrm{~s}$ \\
2453247.4346 & $\pm 0.0007 \mathrm{~s}$ \\
2453285.326 & $\pm 0.002 \mathrm{p}$ \\
2453286.2657 & $\pm 0.0007 \mathrm{p}$ \\
2453302.2672 & $\pm 0.001 \mathrm{p}$ \\
\hline
\end{tabular}
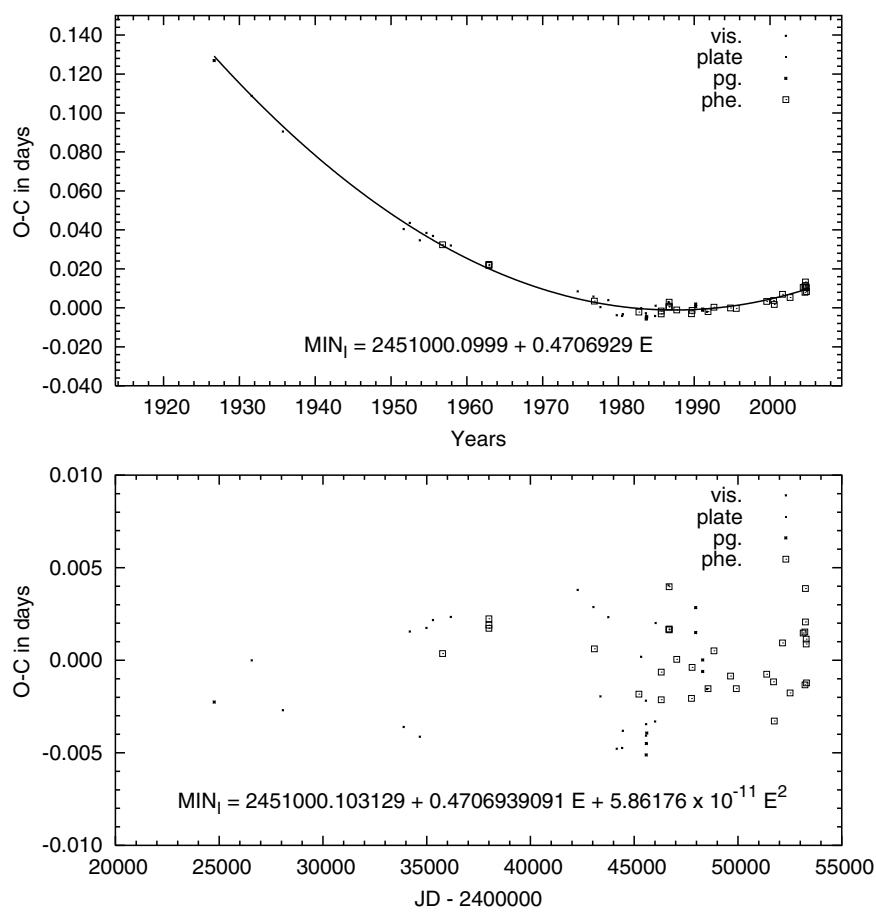

Fig. 6. The O-C curve of the eclipsing binary DK Cyg, with the linear ephemeris taken from Kiss et al. (1999) (above), and the residual curve after subtracting the parabola.

binaries, every 5-10 years by the use of the most recent data points. This is first because, in several cases, it cannot be decided from observations with gaps whether an abrupt period jump has occurred in a given time or if we see some continuous variation. However, a more or less continuous monitoring could soon discover if something interesting is happening, and would lead the observers (nowadays sometimes skilled amateurs with advanced equipment like CCD cameras) to observe the given binary more frequently. Second, returning to this particular system, the constancy of the period variation (at least in the last 80 years), and the lack of any other features from the $\mathrm{O}-\mathrm{C}$ curve needs some explanation, especially because in most cases the $\mathrm{O}-\mathrm{C}$ curves of the contact binaries show more complex behaviour. 


\subsection{V566 Ophiuchi}

This A-type contact binary was discovered by Hoffmeister (1935). Although it was one of the most popular contact binaries during its "first 60 years", it seems to have been neglected in the past decade. V566 Oph did have a stable light curve and constant period in the past (Bookmyer 1976; Eaton 2001). After 1983 the depths of eclipse were increased (Lafta \& Grainger 1985). The behaviour of V566 Oph is not understood, so it requires further study. Due to the light curve changes, the system seems to be a rather active star.

The latest period analysis was carried out by Qian (2001b), who fitted a least-squares parabolic ephemeris and, after subtracting this parabola, he also fitted a pure sinusoidal term, with an amplitude of $0.0037 \mathrm{~d}$ and a period of $20.4 \mathrm{yr}$. He interpreted this latter component as the trace of a possible third companion.

As already mentioned in Sect. 2, the non-simultaneous fitting of the secular and periodic terms might give inaccurate results. This was the main reason we repeated this earlier $\mathrm{O}-\mathrm{C}$ analysis; the other reason, of course, is that some new minima times have been obtained since then. Our times of minima data cover the interval HJD $2434179-2452854$. As was mentioned earlier, there are only very few recent minima. We only found two within the last 7 years. In the present analysis we omitted the visual minima, because first the total observing interval is satisfactorily covered by photoelectric minima and, second, the number of the visual minima is so small that their statistical significance, which seems to be evident e.g. in the case of AB And or U Peg, fails here.

After omission of four evidently bad photoelectric data, we simultaneously fitted a quadratic polynomial and the Fourierterms up to the first harmonics. The best fit is plotted in Fig. 7, while the calculated LITE orbit parameters are listed in Table 5. Comparing our results with those of Qian (2001b), both the parameters of the parabola and the periodic terms are similar, for two reasons. First, the period of the quasi-sinusoidal term is less than half of the length of the data series; consequently, the periodicity can be calculated almost independently of the quadratic term parameters. Second, the amplitude of this term is small with respect to the secular period change rate. Although in Table 5 we present the LITE solution for this almost 20 -year period modulation, a possible magnetic origin is also investigated in Sect. 4.3.

\subsection{U Pegasi}

This W subtype system is one of the oldest known W UMa type binaries, as was already discovered 110 years ago (Chandler 1895). In a recent study, Djurašević et al. (2001) investigated the long term photometric variations of the system over the time interval of 1950 to 1989 . They concluded that the complex variations of the light-curves during these four decades can be explained by the variable spot activity of the cooler component. The latest period study was carried out by Pribulla \& Vaňko (2002), who analysed the features of the O-C diagram between 1894 and 2001. These authors explained the centurylong period decrease by conservative mass transfer from the more massive to the less massive component. Furthermore,
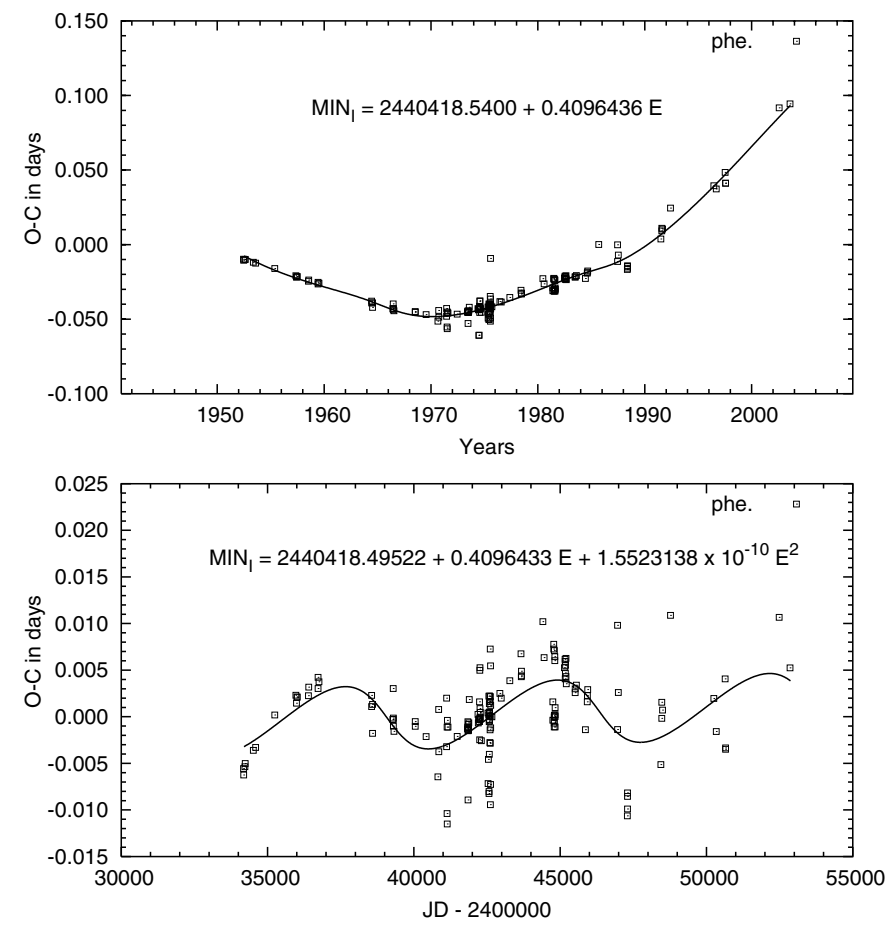

Fig. 7. The O-C curve of the eclipsing binary V566 Oph, with the linear ephemeris taken from the GCVS (Kholopov et al. 1998) (above), and the LITE fit on the parabola-subtracted curve. The quadratic and Fourier-fits were carried out simultaneously.

Table 5. LITE solution for V566 Oph.

\begin{tabular}{|c|c|c|}
\hline \multicolumn{3}{|c|}{$\mathrm{MIN}_{\mathrm{I}}=2440418.49522(7)+0.4096433(22) E+1.55(11) \times 10^{-10} E^{2}$} \\
\hline$P^{\prime}$ & (day) & 7250 \\
\hline$e^{\prime}$ & & $0.31(8)$ \\
\hline$\omega^{\prime}$ & $\left({ }^{\circ}\right)$ & $180(14)$ \\
\hline$\tau^{\prime}$ & (HJD) & $2453590(267)$ \\
\hline$a_{12} \sin i^{\prime}$ & $\mathrm{AU}$ & $0.63(5)$ \\
\hline$f\left(m_{3}\right)$ & $M_{\odot}$ & $0.00065(13)$ \\
\hline$K_{12}$ & $\mathrm{~km} \mathrm{~s}^{-1}$ & 1.00 \\
\hline \multirow[t]{3}{*}{$m_{3}\left(M_{\odot}\right)$} & $i^{\prime}=90^{\circ}$ & 0.14 \\
\hline & $i^{\prime}=60^{\circ}$ & 0.17 \\
\hline & $i^{\prime}=30^{\circ}$ & 0.30 \\
\hline
\end{tabular}

they found a short period $\left(P_{\text {mod }}=18.8 \pm 3.9\right.$ years $)$, low amplitude oscillation. This feature had also been reported earlier by Zhai et al. (1984).

Our O-C data cover 39856 days between HJD 2413094 (1894) and 2452950 (2003). These data are plotted in the upper panel of Fig. 8. We carried out our analysis in a similar way as the one described in the case of $\mathrm{AB}$ And; i.e. we fitted simultaneously a quadratic polynomial and two fundamental frequencies allowing the first harmonic at the longer period as well. Our best fit gave the following two periods: $P_{1} \approx 31059$ days, $P_{2} \approx 6544$ days. If the first period belongs to a light-time orbit due to the existence of an additional star in a hierarchical triple (multiple) system the corresponding orbital 

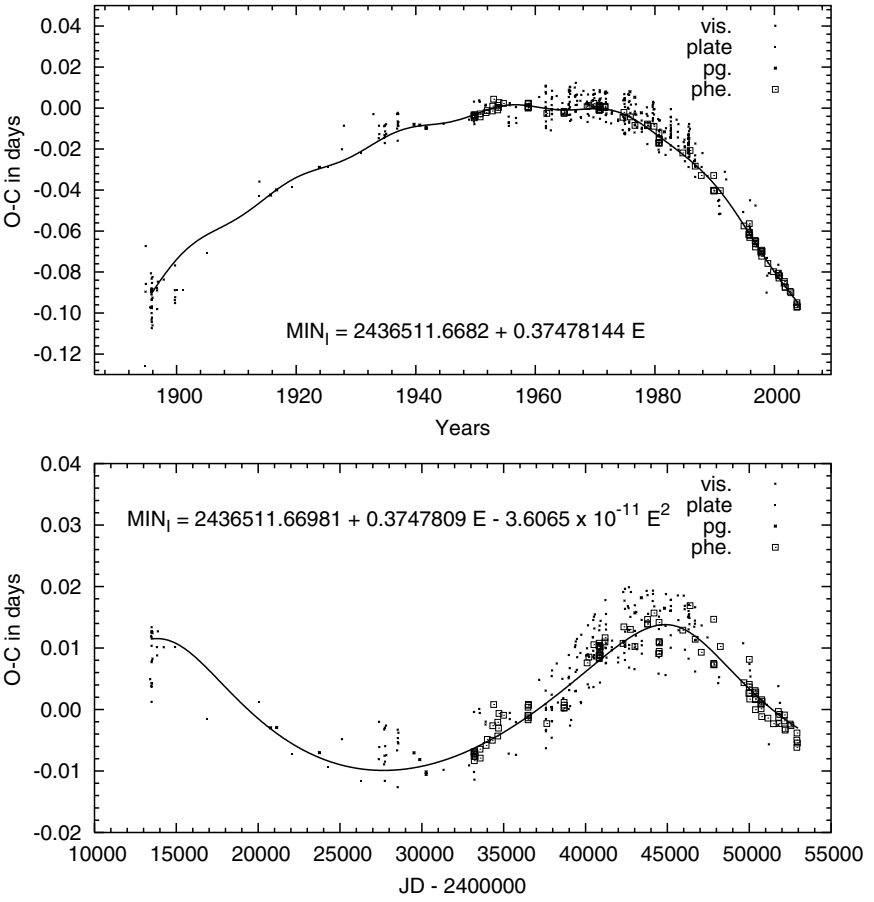

Fig. 8. The O-C curve of the eclipsing binary U Peg with our final solution, plotted against the linear ephemeris taken from the GCVS (Kholopov et al. 1998) (above), and the pure LITE fit on the parabola subtracted curve. The quadratic and Fourier-fits were carried out simultaneously.

elements and derived quantities can be read in Table 6. The latter period is approximately the same as that reported in the previous papers; what perhaps is more interesting, this is also very similar to the length of the modulations in the previous stars. Nevertheless, in the present case it is questionable that this is a real period variation. As can be seen well in Fig. 9, the total amplitude of this "variation" is only $A_{2} \approx 0.003$, i.e. smaller than the scatter of the photoelectric minima, which is usual in W UMa binaries, as a consequence of the varying light-curve shapes distorted by spots (see e.g. Kalimeris et al. 2002). In our opinion it might be imagined that the presence of such a periodicity, especially between 1960 and 1980, is only a result of the small number of photoelectric measurements. However, the fact that similar periodicity was found in the other cases, too, makes it reasonable that this feature might be real.

\section{Discussion}

The five investigated systems are distributed in the range of effective temperature from $T_{\text {eff }}=7400$ down to $T_{\text {eff }}=5400$, which is the transition region where a convection zone develops in the envelope of the stars toward the lower temperatures. Analysis of the $\mathrm{O}-\mathrm{C}$ diagrams of the systems has revealed different periodic variations in four cases besides the secular development of the orbital period.

\subsection{Secular period changes}

Four of the five $\mathrm{O}-\mathrm{C}$ curves show evidence of continuous orbital period change with a constant rate during the total
Table 6. LITE solution for U Peg.

\begin{tabular}{lll}
\hline \hline \multicolumn{2}{l}{$\mathrm{MIN}_{\mathrm{I}}=$} & $2436511.6698(5)+0.3747809(5) E-3.61(10) \times 10^{-11} E^{2}$ \\
\hline$P^{\prime}$ & (day) & 31059 \\
$e^{\prime}$ & & $0.38(5)$ \\
$\omega^{\prime}$ & $\left.{ }^{\circ}\right)$ & $112(9)$ \\
$\tau^{\prime}$ & $(\mathrm{HJD})$ & $2445935(569)$ \\
$a_{12} \sin i^{\prime}$ & $\mathrm{AU}$ & $1.96(13)$ \\
$f\left(m_{3}\right)$ & $M_{\odot}$ & $0.00105(20)$ \\
$K_{12}$ & $\mathrm{~km} \mathrm{~s}^{-1}$ & 0.74 \\
\hline$m_{3}\left(M_{\odot}\right)$ & $i^{\prime}=90^{\circ}$ & 0.14 \\
& $i^{\prime}=60^{\circ}$ & 0.17 \\
& $i^{\prime}=30^{\circ}$ & 0.30 \\
\hline
\end{tabular}

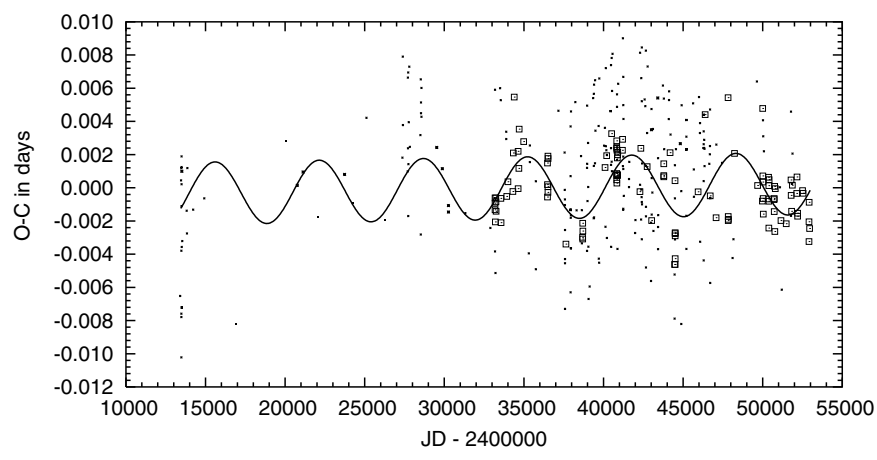

Fig. 9. The shorter period sinusoidal fit on the $\mathrm{O}-\mathrm{C}$ of $\mathrm{U}$ Peg. For the sake of clarity, we subtracted the parabola, as well as the longer period LITE solution, from the original curve.

observing interval. The only exception was OO Aql, although it is possible that in this system only one (or two) abrupt period jump(s) obscures this behaviour.

The most usual explanation of this kind of secular period variation is mass exchange in the system. The widely used approximating formula for the calculation of the mass exchange rate is as follows:

$\dot{m}=-m_{12} \frac{q}{1-q^{2}} \frac{\dot{P}}{3 P} \approx-m_{12} \frac{q}{1-q^{2}} \frac{2}{3} \frac{c_{2}}{c_{1}^{2}}$,

where $c_{1}, c_{2}$ directly come from the $\mathrm{O}-\mathrm{C}$ ephemerides in the form of Eq. (1). Note that the equation requires constancy of the total mass, as well as of the total angular momentum. The rate of the secular period change, as well as the calculated mass exchange rate, are tabulated in Table 7 .

Nevertheless, it is necessary to note, that in the case of $\mathrm{AB}$ And and $\mathrm{U}$ Peg, the coefficients of the quadratic terms and the period and amplitude of the longer period quasisinusoidal period variations are not independent of each other as we will see later.

\subsection{Longer period cyclic variations}

In three cases the simultaneous fitting gave longer, as well as shorter, period cycles. These longer scale variations are roughly 62,75 , and 85 years long and naturally could be identified as a LITE, i.e. effect due to the presence of a third body. 
Table 7. Derived parameters from the period variations.

\begin{tabular}{lllll}
\hline \hline Name & $\dot{P}_{\text {sec }} / P$ & $|\dot{m}|$ & $P_{\text {mod }}$ & $A_{\text {mod }}$ \\
\hline & $\times 10^{-7}\left(\mathrm{yr}^{-1}\right)$ & $\times 10^{-7}\left(M_{\odot} \mathrm{yr}^{-1}\right)$ & $(\mathrm{d})$ & $(\mathrm{d})$ \\
\hline AB And & 2.65 & 1.25 & 6695 & 0.0020 \\
OO Aql & - & - & 7467 & 0.0036 \\
DK Cyg & 1.93 & 0.49 & - & - \\
V566 Oph & 6.76 & 1.25 & 7250 & 0.0035 \\
U Peg & -1.88 & 0.36 & 6544 & 0.0016 \\
\hline
\end{tabular}

The corresponding LITE solution parameters were given in the corresponding tables along with the detailed discussion of the systems. The identification of these periods as LITE solution can be considered only as a simple designation, and their real origin should still require a careful analysis of all cases.

It should also be emphasised that these periods are in the same order as the total interval of the observations, and the separation of the different effects in the analysis are doubtful. For example, let us consider e.g. the case of AB And. In this case the fundamental Fourier-terms are as follows:

$f=0.015 \sin \left(9.17 \times 10^{-5} E\right)-0.017 \cos \left(9.17 \times 10^{-5} E\right)+\ldots$,

which can be written as

$f=-0.017+1.38 \times 10^{-6} E+7.15 \times 10^{-11} E^{2}+\ldots$,

where as seen well, the quadratic term is in the same order of magnitude than the coefficient of the parabolic one. This fact is critical mainly in these two above mentioned cases ( $\mathrm{AB}$ And and $U$ Peg), as in both systems only the last half quasi-sines are covered well by good-quality photoelectric data. On the other hand, despite this fact, we can stress that at least the order of magnitude of the calculated secular period change is believed to be correct.

\subsection{Shorter period cyclic variations - manifestation of magnetic activity cycles?}

It is an interesting fact that four of our five systems show small amplitude cyclic fluctuations with very similar periods (see Cols. 4-5 in Table 7). The only exception is DK Cyg, the hottest in the sample, with A8 spectral-type components, whilst the other four are later than F0 down to G5.

These modulation periods range from 6500 to 7500 days (18-20 years), which are quite similar to those observed in other types of stars showing magnetic activity. Comparing them to the longer period components of the detected variations, where the observations cover only partly a cycle, these shorter cycles are well-covered (several times, 2-4 cycles), as can be seen from the discussion of the individual systems. Hence, the existence and characteristics of these cyclical variations in the orbital period are well proved independently from any doubts about the other - longer period - components.

The startling resemblance of orbital period variations in these more or less similar systems suggests some parallel in their origins and makes some common physical explanations likely. In our opinion this feature might be indirect evidence of similar magnetic cycles in the investigated binaries, and the detected variations might be caused by it.

We mainly refer to Lanza \& Rodonó (1999), who found a relation between the orbital period of a binary system and the magnetic activity cycle by supposing synchronisation between the orbital motion and rotation of the member stars:

$\log P_{\text {mod }}[\mathrm{yr}]=0.018-0.36( \pm 0.10) \log \frac{2 \pi}{P_{\text {orb }}}[\mathrm{s}]$.

For the binaries studied here, this formula predicts $P_{\bmod } \approx$ 7900, 9200, 8500, and 8200 days, respectively (note values very close to the length of the Sun's magnetic cycle). These values are on the same order of magnitude as the observed ones. This supports our conjecture that magnetic activity cycles could explain the observed short term orbital period variations. Furthermore, beyond our sample, Awadalla et al. (2004) reported a similar periodicity of $P_{\bmod } \approx 6500$ days in the O-C curve of the late-type (F8) W UMa binary AK Her, giving additional support to the explanation above.

The rate of this period variation can be expressed easily (see e.g. Applegate 1992) as

$\frac{\Delta P}{P}=2 \pi \frac{A_{\mathrm{O}-\mathrm{C}}}{P_{\bmod }}$

where $\Delta P / P$ is the relative period variation, $A_{\mathrm{O}-\mathrm{C}}$ the halfamplitude of the $\mathrm{O}-\mathrm{C}$ variation, and $P_{\text {mod }}$ the cycle length of the magnetic activity.

In the case of AB And, applying $P_{\text {mod }}=6700$ days and $A=0.002$ days, respectively, we get $\Delta P / P=1.9 \times 10^{-6} \mathrm{~d} / \mathrm{d}$. The variation of the gravitational quadrupole momentum is (Applegate 1992):

$\Delta Q=-\frac{1}{9} M R^{2}(R / a)^{-2} \frac{\Delta P}{P}$

where $M$ is the mass of the active star, $R$ the radius of the active star, and $a=2 A$ the separation between the components. $A$ is the semi-major axis, and note that in contact binaries the orbit is circular. A calculation with the parameters of the primary and the secondary (see Table 1 ) gives $\Delta Q_{1}=4.02 \times 10^{42} \mathrm{~kg} \mathrm{~m}^{2}$ and $\Delta Q_{2}=6.97 \times 10^{42} \mathrm{~kg} \mathrm{~m}^{2}$, respectively. These values correspond to the typical values in active binary stars (Lanza \& Rodonó 1999). Due to the similar absolute dimensions of the other investigated binaries, the same calculations give physically realistic results for them as well.

Nevertheless, we also have to emphasise that in a recent study Qian (2003) did not find clear evidence of decades time-scale period variations in similar systems. $\mathrm{He}$ investigated the period variation of five contact binaries (GW Cep (G3), VY Cet (G5V), V700 Cyg (G2V), EM Lac (G8V), AW Vir (G0V)) and found a secular period change in every case, and he discovered that only two of them show cyclic period variations (VY Cet $\left(P_{3}=7.3\right.$ years), V700 Cyg $\left(P_{3}=39.8\right.$ years $\left.)\right)$. It is an interesting fact that only 40 percent of these $\mathrm{G}$ spectral type systems have cyclic period variations, despite expecting such a phenomenon in all cases. Although both samples (that of Qian 2003 and our) are small, and therefore not representative, the controversion should be 
studied. Our own opinion is as follows. The O-C diagrams of the three remaining systems (GW Cep, EM Lac and AW Vir) consist in only a few points, and there are large gaps between the data. This is due to the fact that these systems were underobserved in the past; therefore, the present observational material is enough to detect the secular period variation only in spite of applying all available minima. Gaps and scattered observations (like visual and photographic ones) obstruct the demonstration of such cyclic variations.

As mentioned already, the spectral type of the systems is later than F0, where we have found short period variations in the $\mathrm{O}-\mathrm{C}$ diagrams, the earlier type DK Cyg does not show this feature. This type of behaviour is pretty similar to the observed one among the Algol-type RS CVn systems (see Hall 1989) so that the magnetic activity can be observed only in systems later than F5. Because the magnetic dynamo theories are based on a strong connection with the presence of a convection zone in the outer envelope predicting magnetic activity for them. Since the convection zones in the envelope appear in lower mass MS and cooler stars, this type of relation is a trivial requirement but, of course, the strict border lines can be different. Although our sample is not a systematic one, it indicates the existence of a separation border somewhere around the spectral type F0.

\section{Conclusions}

The orbital period is very sensitive to any effect and it could be measured with very high precision down to $10^{-9}$ part of the period, and we have a lot of series of minimum observations, in some cases back to the 19th century. In this study we have investigated the $\mathrm{O}-\mathrm{C}$ diagrams of five $\mathrm{W}$ UMa systems from A8 to G5 spectral type components. In four cases a short time scale ( $\simeq 20$ years) cyclic variation of the orbital period was found. All these systems contain later than F0 spectral-type components, which means that they have deep convection zones. As we showed the very similar modulation periods that were observed seem to be independent of any LITE caused by a third body, but a consistent explanation of their cause might also be a magnetic activity cycle.

The mechanism proposed by Applegate (1992) seems to be responsible for the observed variations, and the investigation of close binary systems from this aspect could be an important tool for studying the magnetic properties of stars in general. However, contact binary stars are generally faint and the geometry very complex, so that it is very difficult to apply these methods until further further developments (see e.g. Hendry \& Mochnacki 2000).

Of course, these studies have to be complemented by detailed analyses of the systems from other viewpoints, e.g. observations of the time variable features of their spectra, modelling of time development of their light curves, etc. For example, to check our hypothesis about the magnetic activity, we should follow in time the spotted light curve models of the systems determining their spottedness-index and its correlation with the observed $\mathrm{O}-\mathrm{C}$ variations. These works are in progress and will be published in due time.
Acknowledgements. This research made use of NASA's Astrophysics Data System Abstract and Article Service. This work was partly supported by the Hungarian OTKA Grants T 034551 and T 042509. We thank Dr L. Szabados for his kind help and advice during the preparation of the manuscript.

\section{References}

Applegate, J. H. 1992, ApJ, 385, 621

Awadalla, N., Chochol, D., Hanna, M., \& Pribulla, T. 2004, Contrib. Astron. Obs. Skalnaté Pleso, 34, 20

Baran, A., Zola, S., Rucinski, S. M., et al. 2004, AcA, 54, 195

Barnes, J. R., Lister, T. A., Hilditch, R. W., \& Collier Cameron, A. 2004, MNRAS, 348, 1321

Bookmyer, B. B. 1976, PASP, 88, 473

Borkovits, T., \& Hegedüs, T. 1996, A\&AS, 120, 63

Borkovits, T., Csizmadia, Sz., Hegedüs, T., et al. 2002, A\&A, 392, 895

Borkovits, T., Érdi, B., Forgács-Dajka, E., \& Kovács, T. 2003, A\&A, 398, 1091

Chandler, S. C. 1895, AJ, 15, 185

Csizmadia, Sz., Patkós, L., Moór, A., \& Könyves, V. 2004, A\&A, 417, 745

Demircan, O., Derman, E., \& Akalin, A. 1991, AJ, 101, 201

Demircan, O., \& Gürol, B. 1996, A\&AS, 115, 333

Djurašević, G., \& Erkapić, S. 1999, Ap\&SS, 262, 305

Djurašević, G., Rovithis-Livaniou, H., \& Rovithis, P. 2000, A\&A, 364, 543

Djurašević, G., Rovithis-Livaniou, H., Rovithis, P., Erkapić, S., \& Milovanović, N. 2001, A\&A, 367, 840

Eaton, J. A. 1986, AcA, 36, 275

Guthnick, P., \& Prager, R. 1927, AN, 229, 455

Hall, D. S. 1989, Space Sci. Rev., 50, 219

Hazlehurst, J. 2001, The Obs., 121, 86

Heintz, W. D. 1993, PASP, 105, 586

Hendry, P. D., \& Mochnacki, S. W. 1998, ApJ, 504, 978

Hendry, P. D., \& Mochnacki, S. W. 2000, ApJ, 531, 467

Hershey, J. L. 1975, AJ, 80, 662

Hoffleit, D. 1932, Harvard Obs. Bull, 807, 9

Hoffmeister, C. 1935, AN, 255, 401

Hrivnak, B. J. 1988, ApJ, 335, 319

Hrivnak, B. J. 1989, ApJ, 340, 458

Hrivnak, B. J., Guinan, E. F., \& Lu, W. 1995, ApJ, 455, 300

Hrivnak, B. J., Guinan, E. F., DeWarf, L. E., \& Ribas, I. 2001, AJ, 121,1084

Kalimeris, A., Rovithis-Livaniou, H., Rovithis, P., et al. 1994, A\&A, 291, 765

Kalimeris, A., Rovithis-Livaniou, H., Rovithis, P., et al. 2002, A\&A, 387, 969

Kaszás, G., Vinkó, J., Szatmáry, K., et al. 1998, A\&A, 331, 231

Kähler, H. 1986, MitAG, 67, 85

Kähler, H. 2004, A\&A, 414, 317

Kholopov, P. N., Samus, N. N., Frolov, M. S., et al. 1998, Combined General Catalogue of Variable Stars, 4.1

Kiss, L. L., Kaszás, G., Fűrész, G., \& Vinkó, J. 1999, IBVS, 4681

Kopal, Z. 1978, Dynamics of Close Binary Systems (Dordrecht: D. Reidel)

Kreiner, J. M. 1977, The Interaction of Variable Stars with their Environment, held in Bamberg, September 6-9, 1977, Bamberg: Remeis-Sternwarte, edited by Rudolf Kippenhahn, J. Rahe, and W. Strohmeier., Proc. IAU Colloq., 42, 393

Kreiner, J. M., Rucinski, S. M., Zola, S., et al. 2003, A\&A, 412, 465

Lafta, S. J., \& Grainger, J. F. 1985, Ap\&SS, 114, 23 
Lanza, A. F., \& Rodonó, M. 1999, A\&A, 349, 887

Lanza, A. F., \& Rodonó, M. 2002, AN, 323, 424

Lubow, S. H., \& Shu, F. H. 1977, ApJ, 216, 517

Lucy, L. B. 1976, ApJ, 205, 208

Maceroni, C., \& van't Veer, F. 1996, A\&A, 311, 523

Maceroni, C., Vilhu, O., van't Veer, F., \& van Hamme, W. 1994, A\&A, 288, 529

Mochnacki 1981, ApJ, 245, 650

Niarchos, P. G., Rovithis-Livaniou, H., \& Rovithis, P. 1993, Ap\&SS, 203, 197

Paparó, M., Hamdy, M. A., \& Jankovics, I. 1985, IBVS, No. 2838

Pribulla, T., \& Vaňko, M. 2002, Contrib. Astron. Obs. Skalnaté Pleso, 32,79
Pych, W., Rucinski, S. M., DeBond, H., et al. 2004, AJ, 127, 1712

Qian, S. 2001a, MNRAS, 328, 635

Qian, S. 2001b, MNRAS, 328, 914

Qian, S. 2003, MNRAS, 342, 1260

Rovithis-Livaniou, H., Kranidiotis, A., Fragoulopoulou, E., Sergis, N., \& Rovithis, P. 1999, IBVS, 4713

Stepien, K., Schmitt, J. H. M. M., \& Voges, W. 2001, A\&A, 370, 157 van't Veer, F. 1979, A\&A, 80, 287

van't Veer, F., \& Maceroni, C. 1989, A\&A, 220, 128

Webbink, R. F. 1976, ApJ, 209, 829

Wolf, M., Molik, P., Hornoch, K., \& Sarounová, L. 2000, A\&AS, 147, 243

Zhai, D.-S., Leung, K.-C., \& Zhang, R.-X. 1984, A\&AS, 57, 487 